INPLASY

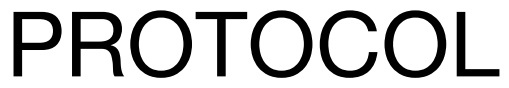

To cite: Shi et al. Quality of evidence supporting the role of YiQi-HuoXue method for the treatment of diabetic kidney disease: A protocol for an overview of systematic review and meta-analysis. Inplasy protocol 202210024. doi: 10.37766/inplasy2022.1.0024

Received: 06 January 2022

Published: 06 January 2022

Corresponding author: Mei Li

limeitcm@163.com

Author Affiliation:

The Second Affiliated Hospital of Shandong University of Traditional Chinese Medicine.

Support: National Natural Science china.

Review Stage at time of this submission: Preliminary searches.

Conflicts of interest: None declared.

\section{Quality of evidence supporting the role of YiQi-HuoXue method for the treatment of diabetic kidney disease: A protocol for an overview of systematic review and meta-analysis}

$$
\text { Shi, HS1; Lv, LN2; Li, YH33; Peng, M4; Si, GM5; Li, M66. }
$$

Review question / Objective: 2.2.1. Type of Studies - The SRs/ MAs of randomized controlled trials (RCTs) based on the method of YQHX for the treatment of DKD were included. Duplicated papers, graduation papers, conference abstracts with insufficient review data, and non-rigorous SRs/MAs are excluded. 2.2.2 Type of Participants - Participants of SRs/MAs are all in compliance with the clinical practice guidelines (K/ DOQI) issued by the National Kidney Foundation(NKF); DKD staging uses the internationally accepted Mogensen diagnostic criteria. There are no restrictions on the recruitment of participants based on gender, age, race, duration, or disease stage. 2.2.3. Type of Interventions - Regarding intervention measures, we divided into two plans: a) The control group was treated with routine treatment (RT), and the intervention group was treated with YQHX method based on the control group; b) the control group was treated with $\mathrm{RT}$, and the intervention group was treated with YQHX method. RT includes basic treatments such as health education, hypoglycemia, blood pressure reduction, and regulation of blood lipid levels, including RAAS inhibitors. 2.2.4. Outcome Indicators - SRs/MAs should have at least one clear result, such as total effective rate, urinary microprotein excretion rate (UAER), urine microalbumin(UM), 24h urine protein quantification (24h UPQ), urea nitrogen (BUN), blood creatinine (Scr), cholesterol (TC), triglycerides (TG), highdensity lipoprotein (HDL-C), low-density lipoprotein (LDL-C), Creactive protein (CRP), glycosylated hemoglobin (HbAlc) or fasting blood glucose (FBG).

INPLASY registration number: This protocol was registered with the International Platform of Registered Systematic Review and Meta-Analysis Protocols (INPLASY) on 06 January 2022 and was last updated on 06 January 2022 (registration number INPLASY202210024).

\section{INTRODUCTION}

Review question / Objective: 2.2.1. Type of Studies - The SRs/MAs of randomized controlled trials (RCTs) based on the method of YQHX for the treatment of DKD were included. Duplicated papers, graduation papers, conference abstracts 
with insufficient review data, and nonrigorous SRs/MAs are excluded. 2.2.2 Type of Participants - Participants of SRs/MAs are all in compliance with the clinical practice guidelines (K/DOQI) issued by the National Kidney Foundation(NKF); DKD staging uses the internationally accepted Mogensen diagnostic criteria. There are no restrictions on the recruitment of participants based on gender, age, race, duration, or disease stage. 2.2.3. Type of Interventions - Regarding intervention measures, we divided into two plans: a) The control group was treated with routine treatment (RT), and the intervention group was treated with YQHX method based on the control group; b) the control group was treated with RT, and the intervention group was treated with YQHX method. RT includes basic treatments such as health education, hypoglycemia, blood pressure reduction, and regulation of blood lipid levels, including RAAS inhibitors. 2.2.4. Outcome Indicators - SRs/MAs should have at least one clear result, such as total effective rate, urinary microprotein excretion rate (UAER), urine microalbumin (UM), 24h urine protein quantification (24h UPQ), urea nitrogen (BUN), blood creatinine (Scr), cholesterol (TC), triglycerides (TG), high-density lipoprotein (HDL-C), lowdensity lipoprotein (LDL-C), C-reactive protein (CRP), glycosylated hemoglobin (HbAlc) or fasting blood glucose (FBG).

Condition being studied: Diabetic kidney disease (DKD) refers to kidney damage caused by diabetes, and proteinuria in diabetic patients is the basic clinical manifestation(1). DKD occurs in approximately one-third of diabetic patients. Early symptoms are not obvious, but as the condition worsens, the rate of deterioration will become faster and faster(2). For decades, it has been the main cause of chronic renal failure in developed countries and has become increasingly popular in developing regions(3). Unfortunately, because diabetes and impaired renal function are closely related to higher mortality and cardiovascular events, DKD has brought a huge economic burden to society(4). It is not difficult to see that effectively preventing and preventing the continuous development of DKD is the focus of the treatment of the disease. Blood glucose management, blood pressure control, and renin-angiotensinaldosterone system (RAAS) inhibitors are currently the main treatments for DKD and have successfully reduced the risk of disease onset or progression(5). Unfortunately, angiotensin-converting enzyme inhibitor(ACEI)/Angiotensin Receptor Blocker(ARB) has side effects such as dry cough, hypotension, hyperkalemia, and angioedema, which limits its scope of application. At the same time, relevant evidence-based studies have shown that these drugs have no obvious control effect on the incidence and mortality of DKD cardiovascular events(6). Some emerging therapies such as sodiumglucose cotransporter-2 (SGLT2) inhibitors (SGLT2is) have been proven to be beneficial for the treatment of DKD, and it has been reported that they can reduce the risk of kidney disease progression by $45 \%(7,8)$. However, SGLT2 inhibitors can cause a series of adverse events such as genital fungal infections, urinary tract infections, hypotension, acute kidney injury, and fractures(9). Although standard treatment can effectively alleviate the progression of DKD, its side effects also affect the lives of patients. Therefore, to improve the quality of life as much as possible, some patients have to seek supplementary and alternative therapies to control their condition. The use of Chinese herbal medicine to treat symptoms similar to DKD can be traced back to the Han Dynasty (B.C.202-A.D.220) (10). In the traditional Chinese medicine system, "qi deficiency" and "blood stasis" are the main pathogenesis of DKD. Therefore, benefiting qi and activating blood circulation are their corresponding basic treatment methods, and it is called the method of "YiQi-HuoXue" (YQHX). The YQHX method based on this theory has been widely used in clinical practice. Relevant studies have confirmed that the method of "YQHX" can improve the renal function of patients with early DKD, reduce urine protein, and have the effect of early intervention in the progression of diabetic nephropathy. The mechanism may be related to the inhibition of blood C-reactive 
protein (CRP), IL-8, and other inflammatory factors in DKD patients, thereby improving the micro-inflammatory state of DKD patients(11). At the same time, YQHX method can also regulate podocyte autophagy and promote the degradation of advanced glycation end products through the mTOR/S6K1/LC3 pathway(12). A systematic review (SRs)/meta-analysis (MAs) is an important tool to guide evidence-based clinical practice and has been widely used in various fields of medicine in recent years. More and more SRs/MAs based on the YQHX method to treat DKD show that traditional Chinese medicine can improve clinical symptoms, reduce various indicators, delay the development of DKD, and improve the quality of life. However, their methodology and the quality of evidence have not been evaluated, and whether their conclusions can provide credible evidence for clinicians is still controversial. The system overview of SRs/MAs is a method that has emerged in recent years. It is used to synthesize multiple SRs/MAs and evaluate their quality and various research results in an attempt to resolve the inconsistencies in them. The purpose of this study is to objectively

\section{METHODS}

Participant or population: Participants of SRs/MAs are all in compliance with the clinical practice guidelines (K/DOQI) issued by the National Kidney Foundation(NKF); DKD staging uses the internationally accepted Mogensen diagnostic criteria. There are no restrictions on the recruitment of participants based on gender, age, race, duration, or disease stage.

Intervention / Comparator: Regarding intervention measures, we divided into two plans: a) The control group was treated with routine treatment (RT), and the intervention group was treated with YQHX method based on the control group; b) the control group was treated with RT, and the intervention group was treated with YQHX method. RT includes basic treatments such as health education, hypoglycemia, blood pressure reduction, and regulation of blood lipid levels, including RAAS inhibitors.
Study designs to be included: The SRs/MAs of randomized controlled trials (RCTs) based on the method of YQHX for the treatment of DKD were included. Duplicated papers, graduation papers, conference abstracts with insufficient review data, and non-rigorous SRs/MAs are excluded.

Eligibility criteria: Participants of SRs/MAs are all in compliance with the clinical practice guidelines (K/DOQI) issued by the National Kidney Foundation(NKF); DKD staging uses the internationally accepted Mogensen diagnostic criteria. There are no restrictions on the recruitment of participants based on gender, age, race, duration, or disease stage.

Information sources: Searched seven electronic databases from their respective establishment time to January 1, 2022, including PubMed, Cochrane Library, EMBASE, China Biomedicine (CBM), China Knowledge Network (CNKI), Wanfang Database, and Chongqing VIP. The language of the publication is not restricted. Document retrieval uses a combination of MeSH terms and free words, such as "diabetic nephropathy", "Yiqihuoxue", "systematic review" and "meta-analysis", and adjusted according to different databases. The search strategy of each database is shown in Table 1.

Main outcome(s): SRs/MAs should have at least one clear result, such as total effective rate, urinary microprotein excretion rate (UAER), urine microalbumin (UM), 24h urine protein quantification (24h UPQ), urea nitrogen (BUN), blood creatinine (Scr), cholesterol (TC), triglycerides (TG), high-density lipoprotein (HDL-C), lowdensity lipoprotein (LDL-C), C-reactive protein (CRP), glycosylated hemoglobin (HbAlc) or fasting blood glucose (FBG).

Quality assessment / Risk of bias analysis / Strategy of data synthesis: Two independent researchers evaluated the methodological quality, report quality, risk of bias, and evidence quality of each SRs/ MAs respectively. The tools used are as follows: Methodological Quality of 
Systematic Reviews 2 (AMSTAR-2)(15), risk of deviation in systematic reviews (ROBIS) (16), preferred reporting project for systematic reviews and meta-analysis (PRISMA)(17), and the classification, evaluation, development, and evaluation of recommendations (GRADE). If there is a disagreement in the process, it will be resolved through discussion or consensus with the third-party reviewer. AMSTAR2 is an SRs/MAs evaluation tool that contains 16 items to evaluate the methodological quality of each included SRs/MAs. To assess the effectiveness of SRs/MAs will be directly affected by seven key items (2, $4,7,9,11,13$, and 15). According to the completion of each item, it can be divided into "No", "Partial Yes" or "Yes". At the same time, the overall confidence of SRs/ MAs results can be divided into four levels: "high", "moderate", "low", and "very low". The ROBIS tool is used to assess the risk of bias of each SRs/MAs. The tool is completed in 3 stages: 1) relevance assessment; 2) assessing some of the issues that may be involved in SRs; 3 ) Evaluating the overall risk of deviation in domain 2 of the interpretation stage. The result was judged as "low", "unclear" or "high". Use the PRISMA checklist to assess the quality of each SRs/MAs report. It has the following areas: (a) title, (b) summary, (c) introduction, (d) method, (e) result, (f) discussion, (g) funding. And it consists of 27 projects. According to the completeness of the project information report, each project is considered "yes" (full report), "partial yes" (partial report), or "no" (no report). The GRADE system classifies the quality of evidence into four levels: "high", "moderate", "low", or "very low", and is used to assess the quality of evidence for each outcome measure registered in these SRs/MAs. If there are research limitations, inconsistencies, inaccuracy, indirectness, or publication bias, the initial score will be reduced(18).

Subgroup analysis: No.

Sensitivity analysis: No.

Country(ies) involved: China.
Keywords: Chinese herbal medicine; YiQiHuoXue method; Systematic review; Metaanalysis; Overview.

Contributions of each author:

Author 1 - Hongshuo Shi.

Author 2 - Lina Lv.

Author 3 - Yinghao Li.

Author 4 - Min Peng.

Author 5 - Guomin Si.

Author 6 - Mei Li. 